\title{
Experiencias de personas con diversidad funcional auditiva y optómetras en la atención en salud visual
}

\author{
Experiences of people with hearing functional diversity and optometrists in visual \\ health care
}

\author{
Leidy Stephanny Abreo Díaz' (D), Angie Melissa Roa Ávila' (D), Diana Paola Maldonado Palacios (D), \\ Gabriela García Landazábal' ${ }^{\text {(D) }}$, Karen Natalia Arias Pineda ${ }^{2}$ (D) María del Pilar Oviedo Cáceres ${ }^{3}$ (D)
} Citación: Oviedo-Cáceres MP, Arias-Pineda KN, García G, Abreo LS, Maldonado DP, Roa AM. Experiencias de personas con diversidad funcional
auditiva y optómetras en la atención en salud visual. Ustasalud 2022; 21(1): 32-41. DOI: https://doi.org/10.15332/us.v21i1.2608

Licencia Creative Commons

1 Estudiante de Optometría, Universidad Santo Tomás, Bucaramanga, Colombia.

2 Comunicadora Social, Universidad Santo Tomás, Bucaramanga, Colombia.

3 Optómetra. PhD en Salud Pública, Universidad Santo Tomás, Bucaramanga, Colombia.

Autor de correspondencia:

María del Pilar Oviedo

Correo electrónico:

maría.oviedo@ustabuca.edu.co

\section{Resumen}

Objetivo: Describir la experiencia de las personas con diversidad funcional auditiva y los profesionales de la salud visual en cuanto las dinámicas que se dan en el interior de la atención en salud. Metodología: Estudio cualitativo descriptivo interpretativo. Se realizaron entrevistas semi-estructuradas vía telefónica, dada la condición de pandemia por la Covid-19, a personas con diversidad funcional auditiva, optómetras e intérpretes de señas. Para el proceso de análisis se utilizó el diseño metodológico desde Taylor y Bogdan. Resultados: A partir del análisis realizado emergen dos categorías: Desconocimiento de los profesionales de la salud sobre los significados de la diversidad funcional auditiva y prácticas positivas para la atención clínica. El desconocimiento de los profesionales se puede explicar por la falta de formación para la atención de la diversidad, lo cual se refleja en la falta de herramientas mínimas por implementar para facilitar la comunicación en el contexto de la atención clínica. Se identifican experiencias positivas en relación con la implementación de algunas prácticas en las que se establecen mecanismos de comunicación en los que se garantiza el derecho a la salud de quienes acuden a la consulta. Conclusiones: Los hallazgos dan cuenta de las barreras para el establecimiento de la comunicación en el ejercicio de la atención optométrica; aun persisten brechas para la garantía de la accesibilidad de las personas con diversidad funcional auditiva.

Palabras clave: Discapacidad auditiva, servicios de salud, barreras de comunicación.

\section{Abstract}

Objective: Describe the experience of people with hearing functional diversity and visual health professionals regarding the dynamics that occur within health care. Methodology: Qualitative interpretive study. Semi-structured interviews were carried out by telephone, given the condition of pandemic by covid-19, to people with hearing functional diversity, optometrists and sign interpreters. For the analysis process, the methodological design from Taylor and Bogdan was used. Results: From the analysis carried out, two categories emerge: Lack of knowledge of health professionals about the meanings of functional auditory diversity and positive practices for clinical care. The lack of knowledge of the professionals can be explained by the lack of training for the care of diversity, which is reflected in the lack of minimum tools to implement to facilitate communication in the context of clinical care. Some positive experiences are identified in relation to the implementation of some practices in which communication mechanisms are carried out in which the right to health of those who attend the consultation is guaranteed. Conclusions: The findings show the barriers to the establishment of communication in the exercise of optometric care; There are still gaps to guarantee accessibility for people with hearing functional diversity.

Keywords: Hearing impairment, health services, communication barriers.
Recibido para publicación: 17 de junio de 2021 Aceptado para publicación: 8 de septiembre de 2021 


\section{INTRODUCCIÓN}

La diversidad funcional auditiva es un término amplio que encierra el conjunto de personas con pérdida auditiva, la cual puede ser tomada como una discapacidad o como una deficiencia, dependiendo el grado de limitación auditiva del individuo [1], generalmente se distinguen dos grupos, entre ellos: sordera, que hace referencia a la pérdida total de la audición e hipoacusia, referente a la pérdida parcial de la capacidad y percepción auditiva, contando con un resto auditivo aprovechable y la posibilidad de mejorar la audición por medio de audífonos electrónicos [2].

Las deficiencias auditivas son clasificadas por la Organización Mundial de la Salud de acuerdo con las recomendaciones emitidas en el 2018 por el grupo de expertos de enfermedades sobre la pérdida auditiva [3]. Actualmente, se clasifica en Leve la persona que percibe en el mejor de sus oídos niveles de sonido entre los 20,0 a 34,9 dB; en este caso, las personas no tienen problemas para escuchar estando en ambientes tranquilos o silenciosos y a su vez pueden tener dificultades para seguir o participar en una conversación en un ambiente ruidoso. Moderada cuando las personas perciben en el mejor de sus oídos niveles de sonido entre los 35-49,9 dB; las personas pueden experimentar en un entorno tranquilo dificultades para escuchar voces en tonos normales y en escenarios ruidosos experimentar dificultades para participar de una conversación. Las personas son capaces de escuchar y repetir palabras con voz alta a 1 metro. Moderada-severa cuando la persona percibe en el mejor de sus oídos niveles de sonido entre los 50-64 $\mathrm{dB}$; en este caso en ambientes silenciosos las personas pueden escuchar un habla fuerte y en ambientes ruidosos experimentar grandes dificultades para participar de una conversación. Severa cuando la persona percibe en el mejor de sus oídos niveles de sonido entre los 65-79,9 dB; en estos casos en escenarios tranquilos las personas pueden escuchar el habla fuerte directamente en el oído y por su parte en escenarios ruidosos una marcada dificultad para participar de una conversación. Profunda cuando las personas perciben, en el mejor de sus oídos niveles de sonido entre los 80 a 94dB; en estos casos las personas tienen grandes dificultades para oír aun en escenarios tranquilos y en aquellos ruidosos no es posible escuchar ningún tipo de conversación. Total: con un nivel de audición de $95 \mathrm{~dB}$ o superior en la cual no se escuchan ningún habla o sonido [3].
La Organización Mundial de la Salud (OMS) en el 2021 estimó que, en el mundo, 1500 millones de personas viven con algún grado de pérdida de audición, de las cuales unos 430 millones necesitan servicios de rehabilitación. Para 2050 está previsto que haya casi 2500 millones de personas con algún grado de pérdida de audición y que al menos 700 millones requieran rehabilitación [4]. En Colombia, y sin datos más recientes, en el 2010, el Departamento Administrativo Nacional de Estadística (DANE) afirma que 159.778 personas son registradas con discapacidad auditiva, de las cuales 8.670 pertenecen al departamento de Santander y 1.035 pertenecen a Bucaramanga [5].

La pérdida auditiva tiene un impacto considerable en la interacción social; la incapacidad o capacidad reducida para comunicarse con amigos y familiares puede afectar las relaciones en el hogar, el trabajo y la escuela. Esto puede tener consecuencias socioeconómicas y también puede afectar el bienestar general [6]. Dentro de la diversa población de personas con discapacidad, las barreras de comunicación son especialmente importantes para las personas sordas o con dificultades auditivas, en este sentido, enfrentan desafíos particulares con respecto a la comunicación con los proveedores de atención médica, y esta es probablemente una razón clave por la cual las personas sordas utilizan los servicios de atención médica de manera diferente a la población general [7]. Informes mencionan que las personas con esta condición ven a los médicos con menos frecuencia, entre otros factores, por la falta de comunicación y la falta de intérpretes disponibles [8].

En el marco del proceso de atención en salud, la interacción del paciente con diversidad funcional auditiva ha generado consecuencias, tales como: la incomodidad al momento de establecer una conversación, dificultando el tiempo de atención en una consulta de optometría. Esto debido a que el personal prestador del servicio no está capacitado para comunicarse adecuadamente con este tipo de usuarios [9]. Los pacientes con discapacidad auditiva han informado que sienten molestias al momento de interaccionar con profesionales de la salud, y a la vez miedo y desconfianza hacia el sistema de salud, estas barreras de comunicación en la prestación de servicios de salud impiden que se realice una valoración efectiva [10]. Así mismo, durante la consulta, se pueden llegar a presentar algunos proble- 
mas, como: el paciente no pueda escuchar las preguntas formuladas, también es posible que el consentimiento informado pueda verse afectado debido a la falta de comprensión total del tratamiento que un profesional de salud le formule. La pérdida auditiva resulta en una incapacidad para acceder al mensaje, lo que deja como consecuencia un déficit de comunicación [11].

Si bien en el contexto internacional se han reportado estudios en relación con las barreras para la comunicación en el marco de una consulta, no se identifican para el caso específico de la atención optométrica, por lo tanto, el presente estudio buscó comprender la experiencia de las personas con diversidad funcional auditiva y los profesionales de la salud visual en cuanto las dinámicas que se dan en el interior de la atención en salud.

\section{MATERIALES Y MÉTODOS}

Se realizó un estudio cualitativo descriptivo interpretativo para acercarse a la descripción de la experiencia de las personas con diversidad funcional auditiva y los profesionales de la salud visual en cuanto las dinámicas que se dan en el interior de la atención en salud. La selección de los participantes fue intencional bajo los criterios de entrevistado especial, definido como aquel que se ubica en una posición única en la comunidad [12]. Para ser considerados en la investigación, las personas debían ser mayores de edad, tener diversidad funcional auditiva, ser interprete de lengua de señas u optómetra.

En la investigación participaron 7 mujeres y 5 hombres con una edad promedio de 39 y 31 años, respectivamente, entre los cuales se encuentran 6 optómetras, 3 pacientes con diversidad funcional auditiva y 3 intérpretes de lengua de señas colombiana. Algunas entrevistas se realizaron de manera presencial, especialmente aquellas de los profesionales en optometría. Sin embargo, y teniendo en cuenta la condición de aislamiento por la pandemia por el brote de la Covid-19, se utilizó como herramienta de recolección de información la entrevista semiestructurada vía telefónica o telemática (Microsoft Teams). Quienes participaron fueron contactados personalmente por las investigadoras, quienes explicaron la intención del estudio y sus alcances. Las entrevistas fueron realizadas con el acompañamiento de las investigadoras $\mathrm{KA}$ y $\mathrm{PO}$, con experiencia en investigación cualitativa y trabajo con personas con discapacidad. Cada entrevista duró en promedio 40 minutos, en los que dos investigadoras siempre estuvieron presentes; mientras que una dirigió la entrevista, la otra tomó notas e incluyó aspectos claves para el análisis.

Las personas participantes del proceso fueron indagadas en relación con sus experiencias cotidianas en las atenciones en salud y, específicamente, en consultas de optometría y oftalmología y las barreras o facilitadores. A las personas con diversidad auditiva se le formularon las siguientes preguntas: ¿Cómo ha sido su experiencia en las consultas de optometría? Desde su experiencia, ¿cuáles son las barreras que se presentan con más frecuencia en la atención de salud visual? ¿Cómo sería posible eliminar dichas barreras? Por su parte a profesionales en optometría se les formularon estas preguntas: ¿Cómo ha sido su experiencia en la atención de pacientes con diversidad funcional auditiva? ¿Qué recursos o materiales conoce para tener en cuenta durante la consulta de optometría de personas con diversidad funcional auditiva? ¿Qué elementos podría implementar en su consultorio para mejorar la atención de las personas con diversidad funcional auditiva? Finalmente a las personas intérpretes se les indagó por ¿cómo ha sido su experiencia como acompañante de una persona con diversidad funcional auditiva en una consulta de optometría? y desde su experiencia, ¿cuáles son las barreras que se presentan con más frecuencia en la atención de salud visual?

Todas las entrevistas fueron grabadas en medio digital a través de una aplicación telefónica o de la plataforma de Microsoft Teams; posteriormente fueron identificadas asignándoseles un código a cada una. Las transcripciones fueron leídas y releídas por los investigadores tomando notas de los pensamientos iniciales y los posibles códigos. Se construyó una matriz de Microsoft Excel con código de colores, realizando de manera independiente una codificación inicial de las entrevistas. Posteriormente, el equipo de investigación revisó y discutió los temas y las citas de apoyo hasta que se alcanzó el consenso para la categorización. Todas las personas invitadas aceptaron participar y posteriormente se les solicitó su consentimiento informado por escrito, para la realización de las entrevistas semies- 
tructuradas. En todo momento se garantizó el manejo ético, anónimo y confidencial de la información y el nombre de quienes participaron.

\section{RESULTADOS}

La experiencia de las personas con diversidad funcional auditiva y de profesionales en optometría estuvo atravesada por barreras que se reflejan, entre otras, en el desconocimiento de los profesionales de la salud sobre la diversidad funcional auditiva y en las dificultades que se viven dadas por la organización del servicio. En la Figura 1 se muestran las categorías emergentes.

El desconocimiento de los profesionales se puede explicar por la falta de formación para la atención de la diversidad, lo cual se refleja en la ausencia de herramientas mínimas a implementar para facilitar la comunicación en el contexto de la atención clínica. Estos aspectos han sido percibidos por los profesionales de la salud y las personas con diversidad funcional auditiva como los elementos centrales que actúan como barreras para la atención adecuada, asertiva y respetuosa, lo cual tiene una implicación directa en la calidad del servicio recibido. Pese a lo anterior, se identifican algunas experiencias positivas en relación con la implementación de algunas prácticas en las que los profesionales, pacientes e intérpretes establecen mecanismos de comunicación en los que se garantiza el derecho a la salud de quienes acuden a la consulta.

\section{Barreras en la atención}

Las personas entrevistadas manifiestan que su experiencia en la atención clínica está marcada por el desconocimiento frente a la discapacidad auditiva y los mecanismos asertivos para la comunicación, entre los que se incluye el lenguaje de señas. Este asunto pasa inicialmente por el mismo significado que los profesionales entrevistados le atribuyen a la condición, pues aún persiste una mirada limitante y un enfoque marcadamente medicalizado.

"Es una alteración de la función auditiva que debe tener el paciente, como hipoacusia o cualquier otro trastorno en su órgano auditivo, que no le permite desarrollar bien esa función" [optómetra].

Sin embargo, desde la perspectiva de las personas entrevistadas con la condición, este asunto enmarca la perspectiva de la diversidad funcional, por lo cual no se entiende como un diagnóstico médico.

Bajo la estética de la medicina somos personas discapacitadas o personas con deficiencia, que es un término que la medicina no debería usar. Nosotros tenemos una diversidad que incluye a todas las personas, porque tú y yo nunca vamos a ser iguales, todos somos diferentes, no hay excepción [persona con diversidad funcional].

Este desconocimiento es una de las principales barreras que se experimentan en la dinámica de la consulta, pues genera obstáculos para la comunicación.

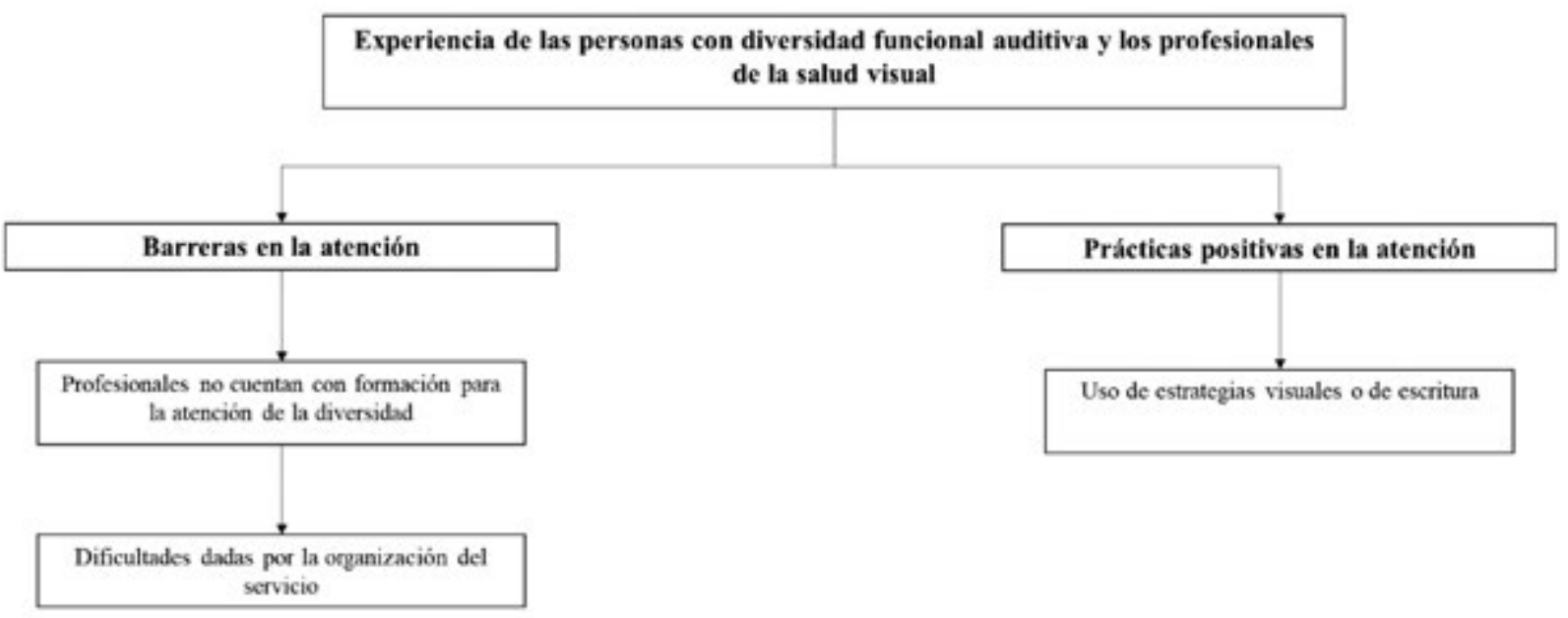

Figura 1. Categorías emergentes. 
Lo anterior, se refleja en testimonios como el siguiente, que dan cuenta de la mirada errónea que tienen los profesionales sobre la manera de establecer el diálogo en la consulta, así como el imaginario de creer que elevando la voz se puede establecer una comunicación más efectiva.

A mí me pasa por lo menos con los sordos, como hay que hablarles duro, que me da pena el tener que gritar porque no quiero que se sienta que lo estoy gritando, entonces me da temor hablarle duro, pero porque me incomoda de pronto lastimar al paciente o a el familiar [optómetra].

Lo anterior a su vez es reconocido por las personas con diversidad funcional e intérpretes

Un doctor empezó a gritar, entonces yo le dije señor grite más o grite menos mi papá no va a oír así de sencillo, el que hablen duro o no, no va a hacer que se entienda ¿sí? sino que se hablen en su mismo idioma y el idioma del sordo es la lengua de señas [intérprete].

Estas barreras generan dificultades tanto para el paciente como para el profesional, para el desarrollo de la atención clínica, por una parte el paciente no encuentra un interlocutor con el cual pueda manifestar sus sentires, necesidades y expectativas y a su vez el profesional no logra identificar adecuadamente los motivos de consulta, interactuar y dar las explicaciones pertinentes sobre la situación de salud visual

"Cuando no está capacitado en lo que implica una discapacidad es más difícil porque es una frustración para el médico y una frustración para el paciente" [intérprete]

Esto a su vez trae como consecuencia el hecho de que los profesionales en ocasiones asuman posturas o gesticulaciones que no son del todo positivas y que nuevamente generan mayores barreras.

Si no entienden se pregunta sin gritar, sin hablar bajito, sin hacer caras, somos personas que aprendemos por la expresión, entonces nosotros miramos algo y somos muy detallistas y nos damos cuenta, no nos gusta que nos traten o hablen como tontos [persona con diversidad funcional].
1.1. Profesionales no cuentan con formación para la atención de la diversidad

Para los profesionales entrevistados, la primera sensación que tienen al saber que alguno de sus pacientes tiene diversidad funcional auditiva es temor o ansiedad, pues reconocen que no tienen la formación para establecer una comunicación adecuada.

Yo recuerdo que lo que hice fue elevar la voz como si eso hiciera que ella pudiera escuchar, esa fue la primera reacción" [optómetra]. Se siente uno un poco de temor, porque no sabe uno cómo se vaya a desenvolver la consulta, que los dos nos podamos entender ¿no?, él para que me cuente qué es lo que le pasa y yo para poderle solucionar su problema. Muchos de nosotros no estamos capacitados en lenguaje de señas [optómetra].

Esta falta de formación de los profesionales genera la necesidad en algunos casos de contar con una persona de apoyo en la consulta, sea un familiar o interprete de señas que acompañe el proceso de atención, lo cual no es un mecanismo que pueda darse fácilmente en todos los casos, pues por un lado, no todos tienen la posibilidad de contar con interprete o acompañante.

El problema es que hay muchos o en su mayoría no manejan la lengua de señas, entonces a veces debo depender de mi mamá, debo depender de algún amigo, pues para que me ayude a interactuar y a comunicarme, a veces cuando no encuentro quién me acompañe, entonces me toca escribir, buscar una palabra adecuada, a veces en estas citas de optometría hay conceptos que uno como persona sorda no maneja o no entiende, entonces es más complicada la comunicación, uno se siente perdido [persona con diversidad funcional].

Estos aspectos son críticos, pues desde la perspectiva de la Convención de los Derechos de las Personas con Discapacidad, se reconoce la autonomía, independencia y autodeterminación de las personas, por lo cual la atención clínica de calidad no debería estar supeditada a un acompañante, pues esto va en contravía de su reconocimiento como sujetos sociales; en este sentido es necesario que los profesionales amplíen su mirada en relación con la identificación de otros mecanismos para comunicarse, como la escritura. 
Para las personas sordas es un poco complejo el hecho de la medicina, porque lo lógico sería que el paciente sordo se comunicara directamente con el profesional de la salud, porque muchas veces son temas muy delicados, muy personales uno quisiera tratar directamente con su médico, para ellos es un poco complejo porque va a haber un tercero ahí [intérprete].

En las dinámicas específicas de la atención clínica optométrica, las personas entrevistadas identifican dificultades para la interacción y la respuesta ante ciertas pruebas clínicas que requieren de una identificación subjetiva por parte del paciente.

Yo no tengo ni idea de hacer señas, cómo le puedo decir ve bien, ve mal, si me escucha o no me escucha, que cara debo hacerla al paciente para que me entienda [optómetra]. La parte subjetiva es fundamental, ahí hay una barrera, me parece complicado pues muchas indicaciones dentro de la consulta como a la hora de hacer una tonometría, una gonioscopia, los procedimientos que sean invasivos, pero la anamnesis me parece tremenda, es difícil lograr captar la idea del paciente [optómetra].

Estas dificultades son percibidas por los pacientes, pues los términos usados, en su mayoría clínicos no son fácilmente reconocidos

“...Hay palabras que no le voy a entender, digamos ipalaras nuevas para mí! y hay personas que hablan muy poquito, muy pasito en la conversación y yo estaría ¿cómo dice?, ¿puede repetirlo? Y más fuerte" [persona con diversidad funcional].

Así mismo, algunas pruebas por la distancia lejana se complejizan en la medida en que no se tiene la certeza sobre si la persona puede seguir leyendo los labios a

"Si uno ya está lejos del paciente, y está al lado del optotipo, hablarle al paciente, pues no sabe si alcanza a leerle a uno los labios, pues bueno eso es un poquito complejo" [optómetra].

De igual manera, algunas prácticas que realizan los profesionales como la escritura en la historia clínica y el poco contacto visual que se mantiene durante la atención son asuntos que aumentan las dificultades.
"La comunicación a distancia también nos afecta mucho si no podemos saber qué están haciendo" [persona con diversidad funcional]. "Normalmente ustedes escriben y no lo miran a uno" [persona con diversidad funcional].

Todos estos aspectos hacen que en ocasiones la consulta se extienda un poco más de lo convencional, generando mayores tensiones para los profesionales pues ellos deben cumplir con una agenda específica.

"Se extiende muchísimo la consulta, ya se hace mucho más larga, entonces ya le coge a uno el afán del tiempo, una cosa la otra, entonces siempre es un poco angustiante" [optómetra].

Uno de los llamados reiterativos que hacen las personas entrevistadas es la necesidad de que el personal de salud tenga inicialmente un proceso de sensibilización en su formación profesional sobre la discapacidad, sus implicaciones y los mecanismos para realizar una consulta inclusiva, para que se respeten los derechos de las personas con discapacidad.

Para tratar de eliminar las barreras de comunicación y actitud de los profesionales de la salud, tendría que hacerse una sensibilización a todo el personal de este sistema de salud sobre las particularidades sociolingüísticas de la comunidad sorda, sus características, para que el profesional entienda que no están tratando con una persona con discapacidad intelectual, porque muchas veces, piensan que por ser sordos tienen comprometida su parte intelectual [intérprete]. Sería que optometría también aprendiera lenguaje de señas, debería ser una obligación que todos los estudiantes aprendieran la diversidad de todos los lenguajes, porque es importante tener el saludo en señas [persona con diversidad funcional].

\subsection{Dificultades dadas por la organización del servicio}

El tránsito por una experiencia de atención en salud inicia desde la misma sala de espera, en la cual los pacientes han experimentado dificultades para identificar el momento en el que son llamados a la consulta, pues la institución no cuenta con un sistema visual que permita acceder a esta información. 
Cuando estamos en sala de espera, que van llamando $\mathrm{y}$ las personas pues escuchan y van ingresando, pero cuando es sordo, uno está ahí como que ¡Dios mío! será que ya me llamó, será que no, a veces uno no entiende y el médico no sabe que es una persona sorda y lo llama y pierde la cita [persona con diversidad funcional].

En el contexto institucional emerge el asunto del intérprete de señas como un apoyo que deberían tener todas las instituciones de salud para la atención; sin embargo, en la clínica donde se realizó este estudio no se cuenta con este servicio, aspecto que debería ser trabajado para garantizar una atención humanizada y de calidad.

Lo primero que le piden al paciente sordo es que venga acompañado de la mamá o del papá o que el mismo consiga y pague un servicio de interpretación cuando debería ser la EPS o el sistema de salud, que le garantice el acceso a la persona sorda, sin la necesidad de que el saque de su bolsillo para pagar un servicio de interpretación [intérprete].

Este panorama ha implicado que el mismo paciente acuda a la institución con una persona que apoye el proceso de atención

El paciente había ido con un acudiente, entonces él me ayudaba un poco a decirme en cuanto a las molestias que tenía el paciente, cuando uno le está haciendo la anamnesis fue el acudiente quien me dio las explicaciones y en el momento de hacer la consulta, pues uno tiene que hacer muchas preguntas, sobre todo en lo subjetivo [optómetra].

Las barreras de comunicación manifestadas por las personas participantes generan una mala percepción sobre los servicios recibidos, lo que a su vez tiene unas implicaciones sobre la adherencia a los tratamientos y sobre la intervención adecuada para el manejo de las alteraciones oculares.

Sabe qué puede pasar a veces también, no es que tengan más dificultad, es que no quieren volverse a enfrentar a una frustración, volver a ir al médico a que no le digan nada, a que le digan vea son estas gafas y resulta que con esas gafas no le funcionan, volverse a enfrentarse a la frustración hace que ellos mismos se retiren [intérprete]. De pronto podrían quedar preguntas sin responder, cosas que el paciente qui- siera saber y no puede expresar o lo expresan y uno no los comprende, el paciente puede quedar con muchas expectativas, con muchas preguntas sin responder [optómetra].

\section{Prácticas positivas en la atención}

Pese al complejo panorama mostrado anteriormente, se identifican prácticas positivas que se constituyen como facilitadores para la atención, tales como el reconocimiento de forma concertada por el paciente y el profesional sobre los mecanismos adecuados para realizar la consulta, ya sea la escritura, el contacto visual permanente para facilitar la lectura de los labios o el contar con un intérprete.

La verdad me ha atendido muy bien, sabe que tengo una condición de vida diferente, no fuera del común, pero sí diferente y ella sabe que como no escucho, me atiende de la forma correcta, entonces me habla sin gritar, me habla de forma correcta me señala, entonces me ha ido muy bien [persona con diversidad funcional]. Me escribe, $o$ me muestra la palabra o me habla sereno hasta que yo entienda, esa es mi experiencia [persona con diversidad funcional].

Estos aspectos son reconocidos por las personas como elementos que facilitaron la consulta y la respuesta ante algunas pruebas específicas, como la toma de la agudeza visual o la repuesta ante la refracción.

Ella empezó a usar la señalización; entonces, me decía de forma concreta tienes que mirar la primera letra, muy fijo con las palabras, y no me confundía, entonces me daba la explicación frente a frente [persona con diversidad funcional]. Cuando ella me dijo que leía los labios, entonces hablar más lento, había como más contacto visual, el hecho de hablarle directamente para hacer más fácil que ella me pudiera ver la cara y pudiera leerme mejor los labios [optómetra].

\section{DISCUSIÓN}

En el contexto internacional, desde la Convención de los Derechos de las Personas con Discapacidad [13] y desde la Ley 1346 de 2009 de Colombia, se exige a los profesionales de la salud que presten a las personas con diversidad funcional, atención de la misma calidad que a las demás, mediante la sensibilización respecto de los 
derechos humanos, la dignidad, la autonomía y las necesidades que puedan tener, a través de la capacitación y la promulgación de normas éticas para la atención de la salud en los ámbitos público y privado. Por su parte, en Colombia y desde la Ley Estatutaria 1618 de 2013 [14], se menciona que las instituciones de salud deben garantizar la accesibilidad e inclusión de las personas con discapacidad en todos sus procedimientos, lugares y servicios; sin embargo, los hallazgos de este estudio permiten identificar la brecha existente para poder dar cumplimiento a esta premisa, pues las personas entrevistadas identificaron como barrera principal la falta de formación del personal de salud para la atención de las personas con discapacidad auditiva y la falta de intérprete de señas en la institución de salud.

La comunicación eficaz es esencial para lograr una atención centrada en el paciente; sin embargo, las barreras de acceso y las suposiciones sobre las preferencias de comunicación pueden generar riesgos que incluyen información médica inexacta e incompleta, errores médicos y diagnósticos erróneos [15]. La falta de formación del personal de salud ya ha sido reconocida por otros estudios previos [16-18]; en cambio esta es la primera investigación rastreada hasta la fecha en el contexto de una atención optométrica, por lo cual los hallazgos de este estudio se consideran elementos valiosos para la reflexión en las facultades o escuelas, pues es necesario que sus estudiantes tengan algún tipo de acercamiento a la temática que les permita en el mediano o largo plazo atender asertivamente a personas con esta condición. Teniendo en cuenta la alta interacción que se da en una consulta entre optómetra-paciente, dada la subjetividad y la necesidad de identificar perfiles visuales o de funcionamiento en algunas de las pruebas clínicas realizadas, es fundamental que la institución de salud incluya herramientas o materiales que favorezcan el ejercicio de la consulta.

Como se menciona en otros estudios de atención en salud general, pocos profesionales de la salud reciben formación sobre cómo comunicarse con las personas con diversidad funcional auditiva; como resultado, los intentos de comunicarse a menudo son inapropiados y se realizan sin conocimiento o comprensión de sus necesidades de comunicación [19].

El apoyo puede ser un requisito previo para participar en algunas personas con discapacidad, con respeto a su dignidad, autonomía e independencia, en este sentido es necesario que las instituciones de salud incluyan en sus procesos administrativos y de calidad las adaptaciones necesarias para que se pueda garantizar el derecho a la salud de este grupo poblacional, tales como el intérprete de señas. Desde la Convención de los Derechos de las Personas con Discapacidad y en Colombia desde la Ley 982 de 2005 se reconoce el derecho a los servicios de apoyo para la comunicación (interpretación en lenguaje de señas); sin embargo, no forman parte casi nunca del marco legislativo, no reciben financiación suficiente, apenas se ofrecen y no corresponden a las necesidades de la gente; este es un aspecto crítico identificado en la presente investigación, pues la institución no cuenta con apoyos para la comunicación, por lo cual es necesario transitar hacia la incorporación de los ajustes razonables que permitan la participación efectiva en sociedad de las personas con discapacidad en igualdad de condiciones con las demás, de todos los derechos humanos y libertades fundamentales.

En esta vía, estudios previos han reconocido la importancia de la presencia de un intérprete en el contexto de una atención en salud, pues permite conocer el motivo de consulta del paciente al profesional y asegurar la exploración y comprensión de los signos y síntomas clínicos; pues las diferencias en la comunicación, y lengua tienen como consecuencia una mala interpretación y errores de diagnóstico [17-20].

Un aspecto crítico que emerge tiene que ver con la necesidad de fortalecer los procesos de formación de los profesionales en optometría sobre lengua de señas colombiana, para favorecer la comunicación efectiva, por tanto, se hace el llamado a la reflexión para que las universidades incluyan en sus planes de estudio espacios académicos que desarrollen esta temática. Tal y como se menciona en otro estudio, para lograr una atención centrada en el paciente, debe existir una comunicación efectiva entre los pacientes y profesionales [21]; sin embargo, los resultados de este estudio dan cuenta de que la comunicación no es la ideal.

Los aspectos mencionados anteriormente tienen una implicación sobre la calidad de la atención que reciben las personas con diversidad funcional auditiva, pues, por un lado, las instituciones de salud no están reconociendo la necesidad de prestar servicios de salud 
incluyentes que respeten la diversidad y, por otro, los profesionales no cuentan con las herramientas para hacerlo. Estos aspectos actúan de manera sumativa y generan barreras que contribuyen en la vulneración del derecho a la salud de las personas con diversidad.

En esta investigación se identificaron algunas prácticas positivas, como la posibilidad de algunos profesionales para identificar caminos conjuntos que permitieran fortalecer el proceso de atención, aspectos que han sido reconocidos por otros estudios en los cuales se menciona como un aspecto clave de la relación médico paciente, la incorporación de gestos, conductas de humanización, solidaridad y sensibilización [18-22]. Pese a lo anterior, es necesario continuar trabajando en los diferentes ámbitos de la atención para que las barreras reportadas en este estudio se puedan abordar, y de esta manera construir una sociedad más equitativa, justa y empática con las personas que viven con esta condición.

\section{Agradecimientos}

El equipo de investigación agradece a las mujeres y hombres con diversidad funcional auditiva participantes del proceso, por su generosidad, tiempo y apertura para compartir sus experiencias.

\section{REFERENCIAS}

[1] Díaz SR, Ferreira MAV. Desde la dis-capacidad hacia la diversidad funcional. Un ejercicio de dis-normalización. Rev Int Sociol 2010;68:289-309. DOI: https://doi. org/10.3989/ris.2008.05.22

[2] Angel Obando FR, Casas Monsegny AM, Gómez Gómez O, et al. Audiología básica. Sección de Publicaciones de la Universidad Nacional de Colombia sede Bogotá, https://repositorio.unal.edu.co/handle/unal/7199 (2006, accessed 15 June 2021).

[3] Olusanya BO, Davis AC, Hoffman HJ. Hearing loss grades and the International classification of functioning, disability and health. Bull World Health Organ 2019;97:725-728.

[4] Haile LM, Kamenov K, Briant PS, et al. Hearing loss prevalence and years lived with disability, 1990-2019: findings from the Global Burden of Disease Study 2019. The Lancet 2021;397:996-1009. DOI: https://doi.org/10.1016/ S0140-6736(21)00516-X
[5] Departamento Administrativo Nacional de Estadística. Censo General 2005: Discapacidad, personas con limitaciones permanentes. https://www.dane.gov.co/files/ censo2005/discapacidad.pdf (2006).

[6] Graydon K, Waterworth C, Miller H, et al. Global burden of hearing impairment and ear disease. J Laryngol Otol 2019;133:18-25. DOI: 10.1017/S0022215118001275

[7] Kritzinger J, Schneider M, Swartz L, et al. 'I just answer "yes" to everything they say': access to health care for deaf people in Worcester, South Africa and the politics of exclusion. Patient Educ Couns 2014;94:379-383. DOI: 10.1016/j.pec.2013.12.006

[8] Lezzoni LI, O'Day BL, Killeen M, et al. Communicating about health care: observations from persons who are deaf or hard of hearing. Ann Intern Med 2004;140:356-362.

[9] Arteaga D, Correa S, Duque k, Ruiz S. Abordaje prehospitalario del paciente con discapacidad auditiva [Especialización]. Universidad CES; 2013.

[10] Newton VE, Shah SR. Improving communication with patients with a hearing impairment. Community Eye Health 2013;26:6-7.

[11] Pakulski LA, McCormick M, Robbins T, et al. Understanding and addressing the impact of hearing acuity on healthcare and healthcare communication. J Interprofessional Educ Pract 2019;15:138-141. DOI: https://doi. org/10.1016/j.xjep.2019.03.003

[12] De la Cuesta C. The quality of qualitative research: from evaluation to attainment. Texto Contexto Enferm 2015;24:883-890. DOI: https://doi. org/10.1590/0104-070720150001150015

[13] Naciones Unidas. Convención sobre los derechos de las personas con discapacidad, https://www.un.org/esa/ socdev/enable/documents/tccconvs.pdf (2006).

[14] Congreso de Colombia. Ley Estatutaria 1618 de 2013, https://www.minsalud.gov.co/sites/rid/Lists/ BibliotecaDigital/RIDE/DE/PS/documento-balance-1618-2013-240517.pdf (2013, accessed 23 April 2019).

[15] Agaronnik N, Campbell EG, Ressalam J, et al. Communicating with Patients with Disability: Perspectives of Practicing Physicians. J Gen Intern Med 2019;34:11391145. DOI: 10.1007/s11606-019-04911-0.

[16] Villamizar AG, Gutiérrez OY, Ruiz LJP. Dificultades y retos del psicólogo clínico en la atención a personas sordas. Rev Elec Psic Izt. 2020;23(4):1612-1629. 
[17] Sanz Lledó AI, García Vallejo R. Percepción de la atención sanitaria recibida en atención primaria por personas con discapacidad auditiva. Rev Rol Enferm 2019;408-412.

[18] Santos AS, Portes AJF. Perceptions of deaf subjects about communication in Primary Health Care. Rev Lat Am Enfermagem 2019;27: e3127. DOI: 10.1590/1518-8345.2612.3127.

[19] Dickson M, Magowan R, Magowan R. Meeting deaf patients' communication needs. Nurs Times 2014;110:12-15.
[20] Barranco Morente S, Ventura Puertos PE, Coronado Carvajal P. Estrategias de comunicación de los profesionales de enfermería con personas sordas o ciegas. Index Enferm 2016;25:253-257.

[21] Hommes RE, Borash AI, Hartwig K, et al. American Sign Language Interpreters Perceptions of Barriers to Healthcare Communication in Deaf and Hard of Hearing Patients. J Community Health 2018;43:956-961. DOI: 10.1007/s10900-018-0511-3

[22] Rodríguez Silva H. La relación médico-paciente. Rev Cuba Salud Pública 2006;32:0-0.

\section{Correo de autores}

Angie Melissa Roa Ávila: angie.roa@ustabuca.edu.co Diana Paola Maldonado Palacios: diana.maldonado@ustabuca.edu.co

Gabriela García Landazábal: gabriela.garcia@ustabuca.edu.co Karen Natalia Arias Pineda: karen.arias01@ustabuca.edu.co María del Pilar Oviedo: maría.oviedo@ustabuca.edu.co 